\title{
Erector Spinae Block for Chest Trauma in Aeromedical Prehospital and Retrieval Medicine
}

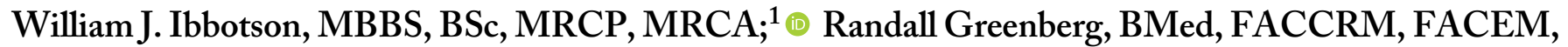 \\ FRACMA; ${ }^{2}$ Peter Brendt, MD, FANZCA, EDIC, DESA ${ }^{3}$
}

1. Medical Officer, Royal Flying Doctor Service South East Section; Advanced Trainee, Department of Anesthesia and Perioperative Medicine, Dubbo Base Hospital, Dubbo, NSW, Australia

2. Chief Medical Officer, Royal Flying Doctor Service South East Section; Director of Critical Care, Dubbo Base Hospital; Associate Professor, School of Rural Health, University of Sydney; State Retrieval Consultant, Ambulance New South Wales, Sydney, NSW, Australia

3. Team Leader Emergency Services, Royal Flying Doctor Service South East Section; Department of Anesthesia and Perioperative Medicine, Dubbo Base Hospital; Department of Emergency Medicine, Sunshine Coast University Hospital, Sunshine Coast, Queensland, Australia

Correspondence:

William J. Ibbotson, MBBS, BSc, MRCP, MRCA

Royal Flying Doctor Service

South Eastern Section

Dubbo Airport

Dubbo, New South Wales, 2830, Australia

E-mail: william.ibbotson@rfdsse.org.au

Conflicts of interest: none

Keywords: anesthesia; chest trauma; erector spinae block; pain; prehospital; retrieval

\section{Abbreviations:}

ESB: erector spinae block

ESM: erector spinae muscle

IV: intravenous

TVP: transverse process

Received: November 12, 2019

Revised: January 2, 2020

Accepted: January 24, 2020

\begin{abstract}
Pain management for patients with chest trauma in aeromedical prehospital and retrieval medicine is important in order to maintain respiratory function. However, it can be challenging to achieve with opioids alone due to side effects including sedation, respiratory depression, and nausea.

Reported are two trauma patients with uncontrolled pain despite multiple doses of opioids managed with a single-injection erector spinae plane block (ESB).

The sono-anatomy and performance of the block, indications, and possible complications associated with the ESB are described.

An ultrasound-guided ESB is useful for multimodal pain therapy following chest trauma in aeromedical retrieval medicine.
\end{abstract}

Ibbotson WJ, Greenberg R, Brendt P. Erector spinae block for chest trauma in aeromedical prehospital and retrieval medicine. Prehosp Disaster Med. 2020;35(4):454-456.

\begin{abstract}
Introduction
The Royal Flying Doctor Service South Eastern (RFDS SE; Sydney, NSW, Australia) provides aeromedical prehospital and retrieval services in rural New South Wales, Australia. Many trauma cases are "primary in-hospital" cases, where the patient is brought from the scene to a remote clinic or hospital. In these remote health care facilities, access to critical care services is limited. Retrieval teams therefore assesses and stabilize the patient locally before transferring them by a combination of road vehicle or fixed wing aircraft (Beechcraft King Air; Textron Aviation, Wichita, Kansas USA) to the receiving center. A common problem in the retrieval environment is pain management. ${ }^{1}$ Thoracic trauma can be very painful, which can impact respiratory function and increase morbidity. ${ }^{2}$ Presented are two cases where erector spinae blocks (ESBs) were successfully used in an aeromedical retrieval setting to control pain in chest trauma.
\end{abstract}

Cases

Written consent to publish their cases was obtained from both patients following their retrieval.

Case 1

A 52-year-old male was brought to a remote clinic following a motor bike accident. He had no significant past medical history and no regular medications. Chest X-ray in the clinic demonstrated a left hemopneumothorax with second to sixth rib fractures and a mid-scapula fracture. His pain score was $8 / 10$ despite multiple doses of morphine, and his breathing was impaired. The patient was not able to lie flat due to pain. An ultrasound-guided ESB was performed by the retrieval team with $30 \mathrm{ml}$ of $0.75 \%$ ropivicaine, followed by the insertion of an intercostal catheter. A total of $40 \mathrm{mg}$ intravenous (IV) ketamine was given to facilitate tube thoracostomy. Following these interventions, the pain score dropped to 1/10 and his breathing pattern improved. The patient was transferred in a lying position via

doi:10.1017/S1049023X20000540

(C) The Author(s), 2020. Published by Cambridge University Press on behalf of the World Association for Disaster and Emergency Medicine. This is an Open Access article, distributed under the terms of the Creative Commons Attribution licence (http://creativecommons.org/licenses/by/4.0/), which permits unrestricted re-use, distribution, and reproduction in any medium, provided the original work is properly cited. 
ambulance and aircraft to the receiving facility and did not require further analgesia for six hours. After arrival at the receiving hospital, pain was controlled with multimodal pain therapy. The patient was discharged from the high dependency unit 48 hours later.

\section{Case 2}

A 24-year-old male presented to a remote clinic having fallen onto a tool box and impaled his chest on a $10 \mathrm{~cm}$ screwdriver. The patient removed the screwdriver himself prior to attending medical services. He had no other injuries. His past medical history included attention deficit hyperactivity disorder (ADHD), cigarette smoking, and daily consumption of cannabis.

Chest X-ray and extended focused assessment with sonography for trauma (eFAST) did not show any significant pathology. Despite treatment with $10 \mathrm{mg}$ morphine IV, $1 \mathrm{~g}$ paracetamol, and $400 \mathrm{mg}$ ibuprofen, the patient was in severe pain $(8 / 10)$ and could not take deep breaths or cough. An ESB was performed under ultrasound guidance with $20 \mathrm{ml}$ of $1 \%$ ropivicaine, following which his pain score reduced to $1-2 / 10$. His breathing pattern improved and he was transported to the receiving hospital via ambulance and aircraft without any further analgesic requirements. The patient was discharged 24 hours later.

\section{Discussion}

The cases presented describe the benefits of regional anesthesia in providing high-quality, opiate-sparing analgesia for prolonged aeromedical retrieval.

The use of regional anesthesia prehospitally has been described for the management of femoral fractures; ${ }^{3}$ and more recently, the use of serratus anterior blocks as analgesia for rib fractures in aeromedical transfer has also been published. ${ }^{4}$ This is the first report in the literature of single-injection ESB being used in the retrieval environment, despite their established use in-hospital. ${ }^{5}$

The ESB is performed with the patient in either the upright sitting or lateral decubitus position, with inline ultrasound guidance. The erector spinae muscle (ESM) complex runs vertically in the back, lateral to the spinal column. It is comprised of the spinale, longissimus thoracis, and iliocostalis muscles. The ESM lies directly on top of the transverse process (TVP) of the vertebral body. The TVP is identified in a longitudinal parasagittal plane approximately $3 \mathrm{~cm}$ lateral to the midline on the affected side using ultrasound (Figure 1). The TVP appears as a flattened, squared acoustic shadow with minimal pleura visible (Figure 2). In contrast, the image of a rib appears rounded with associated pleura underlying (Figure 3). The needle tip is directed onto the TVP and local anesthetic is injected below the ESM on top of the TVP. The anesthetic spreads in a cranio-caudal plane by one dermatome for approximately each $3.4 \mathrm{ml}$ of injectate, ${ }^{6}$ so the target injection site should be in the middle of the area to be blocked. The mechanism of action of the ESB is by anterior diffusion of anesthetic into the paravertebral and epidural spaces, and lateral diffusion into the intercostal spaces. ${ }^{6-8}$ This blocks the dorsal and ventral rami of the spinal nerves, which provide sensation to both the anterior and posterior chest wall.

Complications of the ESB are rare. The block is superficial and performed in an area without significant vascular structures. In-hospital use has been described in coagulopathic patients without complications. ${ }^{9,10}$ The underlying bony anatomy makes identification of the target area easy and protects underlying structures from damage should needle placement be off target. Reported complications include pneumothorax, ${ }^{11}$ incomplete

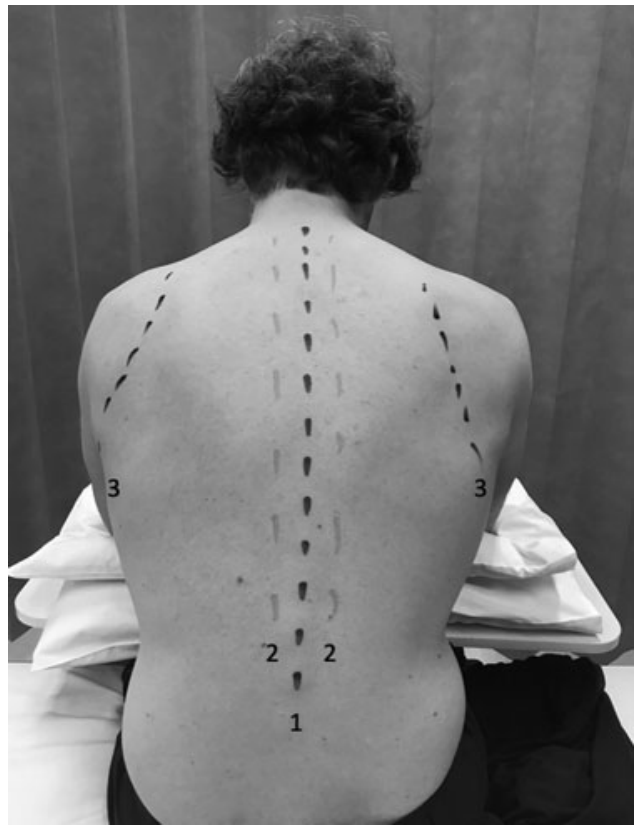

Ibbotson (9) 2020 Prehospital and Disaster Medicine

Figure 1. Positioning of the Patient in the Upright Sitting Position.

Note: 1 = midline/spinous process; 2 = approximate position of the transverse processes; $3=$ position of the scapulae.

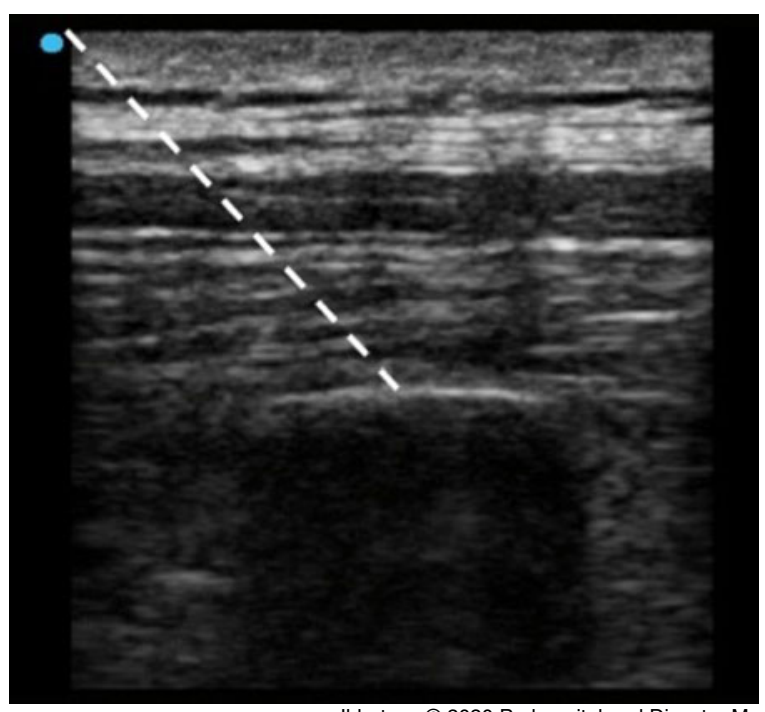

Ibbotson $\odot 2020$ Prehospital and Disaster Medicine

Figure 2. View of the Transverse Process Deep to the Erector Spinae Muscle in the Longitudinal Parasagittal Orientation with a Linear $13-6 \mathrm{MHz}$ Probe.

Note: The transverse process is a square acoustic shadow with no pleura visible. Dotted line: desired needle placement for block.

blockade, ${ }^{12}$ and motor block. ${ }^{13}$ Hematoma is also a theoretical complication, but has not been described in the literature. No complications were reported with either case published.

As with any use of ultrasound, performance of an ESB is an operator-dependent procedure. Different levels of training and clinical background my lead to different challenges and success rates in performing ESBs. Challenges while performing an ESB 


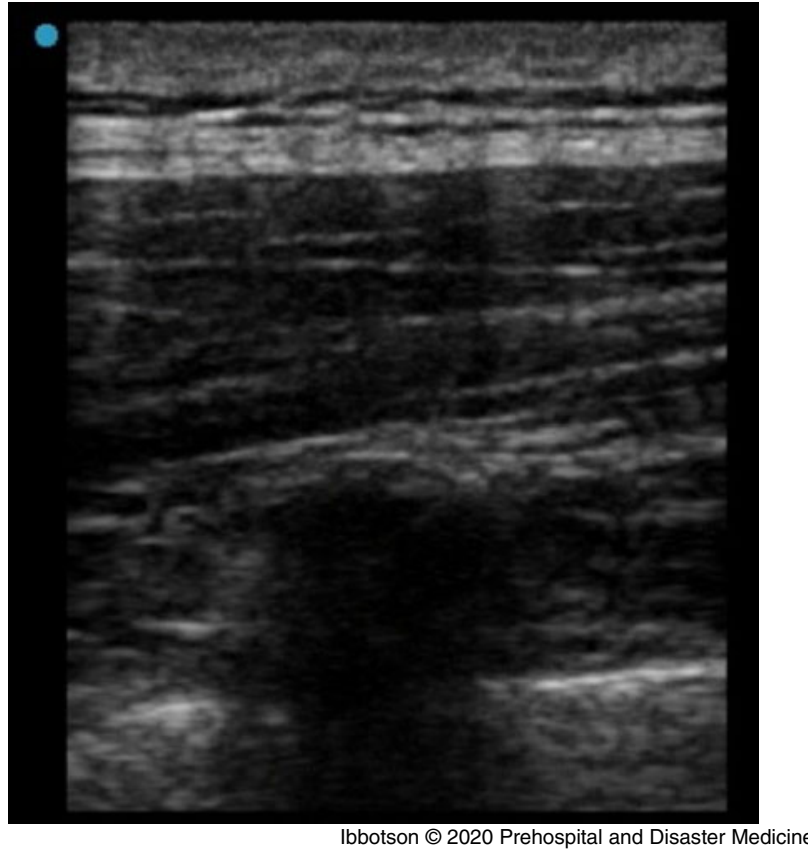

Figure 3. View of a Rib in the Longitudinal Parasagittal Orientation with a Linear $13-6 \mathrm{MHz}$ Probe.

Note: Deep to the erector spinae muscle, the rib appears as a rounded acoustic shadow with underlying pleura.

include: identification of anatomic structures in patients under spinal precautions, or with large body habitus, and maintaining continuous needle visualization.

Other alternative regional anesthetic procedures for rib fractures in-hospital include thoracic epidurals and paravertebral blocks.
However, these blocks are more complex to perform in a retrieval environment, require a greater level of operator skill, are closer to vulnerable anatomic structures, and are contra-indicated in coagulopathic patients.

The use of serratus anterior block for chest trauma ${ }^{14}$ has also been used in retrieval medicine; ${ }^{4}$ however, it lacks coverage of the chest posterior to the mid axillary line ${ }^{15}$ and so may not provide analgesia to posterior rib fractures.

One case report from a military retrieval service reports a multiinjection ESB in a prolonged transfer of a patient with chest trauma. ${ }^{16}$ In contrast, in this case series, a larger injection volume was used to reduce the technique to a single injection.

At the time of publication, no studies have been performed to assess the relative efficacy and safety of myofascial plane blocks (such as the ESB), so it is not possible to ascertain whether the ESB is superior to other forms of regional anesthesia.

\section{Limitations}

The provided data are a retrospective case series. Therefore, generalizing the information to other aeromedical services may be limited due to different settings, methods of transfer, distance of transfer, or level of expertise with ultrasound of the provider. Additional to this, reporting bias by the authors may exist. Therefore, the provided data should only be used to create the hypothesis that ESB are suitable blocks for the management of chest trauma in the aeromedical retrieval setting.

\section{Conclusion}

This case series demonstrate the successful use of single-injection ESBs in the aeromedical retrieval setting as part of multimodal analgesia. Further studies are required to assess the practicability and safety of ESBs in the management of pain from chest trauma during aeromedical retrievals.

\section{References}

1. Lamb D. The documentation of pain management during aeromedical evacuation missions. Nurs Clin North Am. 2010;45(2):249-260.

2. Unsworth A, Curtis K, Asha SE. Treatments for blunt chest trauma and their impact on patient outcomes and health service delivery. Scand J Trauma Resusc Emerg Med. 2015;23:17.

3. Hards M, Brewer A, Bessant G, Lahiri S. Efficacy of prehospital analgesia with fascia iliaca compartment block for femoral bone fractures: a systematic review. Prehosp Disaster Med. 2018;33(3):299-307.

4. McLean J, Cooke S, Burns B, Reid C. First reported helicopter in-flight serratus plane block for rib fractures. Air Medical Journal. 2019;38(5):374-376.

5. Hamilton DL, Manickam B. Erector spinae plane block for pain relief in rib fractures. Br J Anaesth. 2017;118(3):474-475.

6. De Cassai A, Tonetti T. Local anesthetic spread during erector spinae plane block. J Clin Anesth. 2018;48:60-61.

7. Adhikary SD, Bernard S, Lopez H, Chin KJ. Erector spinae plane block versus retrolaminar block: a magnetic resonance imaging and anatomical study. Reg Anesth Pain Med. 2018;43(7):756-762.

8. Ueshima H, Hiroshi O. Spread of local anesthetic solution in the erector spinae plane block. J Clinic Anesth. 2018;45:23.
9. Ayub A, Talawar P, Kumar R, Bhoi D, Singh AY. Erector spinae block a safe, simple and effective analgesic technique for major hepatobiliary surgery with thrombocytopenia. Egyptian J Anaesth. 2019;34(4):169-172.

10. Adhikary SD, Prasad A, Soleimani B, Chin KJ. Continuous erector spinae plane block as an effective analgesic option in anticoagulated patients after left ventricular assist device implantation: a case series. J Cardiothorac Vasc Anesth. 2019;33(4):1063-1067.

11. Ueshima H. Pneumothorax after the erector spinae plane block. J Clin Anesth. 2018; 48:12.

12. Ueshima H, Otake H. Limitations of the Erector Spinae Plane (ESP) block for radical mastectomy. J Clin Anesth. 2018;51:97.

13. Selvi O, Tulgar S. Ultrasound guided erector spinae plane block as a cause of unintended motor block. Revista Española de Anestesiología y Reanimación (English Edition). 2018;65(10):589-592.

14. May L, Hillermann C, Patil S. Rib fracture management. BJA Education. 2016; 16(1):26-32.

15. Durant E, Dixon B, Luftig J, Mantuani D, Herring A. Ultrasound-guided serratus plane block for ED rib fracture pain control. Am J Emerg Med. 2017;35(1):197.

16. Fabich RA, Greene S, Tighe C, Devasahayam R, Becker T. A novel use of the erector spinae block in the austere environment. Mil Med. 2019. 